\title{
@creative
}

\section{Regular dessins d'enfants with field of moduli $\mathbb{Q}(\sqrt[p]{2})$}

\author{
Ruben A. Hidalgo *, Saul Quispe ${ }^{\dagger}$ \\ Departamento de Matemática y Estadística, Universidad de La Frontera, \\ Casilla 54-D, 4780000 Temuco, Chile
}

Received 22 September 2016, accepted 9 December 2016, published online 14 March 2017

\begin{abstract}
Herradon has recently provided an example of a regular dessin d'enfant whose field of moduli is the non-abelian extension $\mathbb{Q}(\sqrt[3]{2})$ answering in this way a question due to Conder, Jones, Streit and Wolfart. In this paper we observe that Herradon's example belongs naturally to an infinite series of such kind of examples; for each prime integer $p \geq 3$ we construct a regular dessin d'enfant whose field of moduli is the non-abelian extension $\mathbb{Q}(\sqrt[p]{2})$; for $p=3$ it coincides with Herradon's example.
\end{abstract}

Keywords: Dessins d'enfants, Riemann surfaces, field of moduli and field of definition.

Math. Subj. Class.: 14H57, 30F10, $11 G 32$

\section{Introduction}

A dessin d'enfant (or hypermap) of genus $g$, as defined by Grothendick in his Esquisse d'un Programme [8], is a bipartite map (vertices come in black and white colors and vertices of the same color are non-adjacent) on a closed orientable surface of genus $g$. The degree of the dessin d'enfant is the number of its edges. As a consequence of the classical uniformization theorem, a dessin d'enfant can also be seen as a pair $(S, \beta)$, where $S$ is a closed Riemann surface and $\beta: S \rightarrow \widehat{\mathbb{C}}$ is a non-constant meromorphic map whose branch values are contained in the set $\{\infty, 0,1\}$; the degree of the dessin is the same as the degree of $\beta$. A dessin d'enfant $(S, \beta)$ is called regular if $\beta$ is a regular branched covering.

The signature of the dessin d'enfant is the tripe $(a, b, c)$, where $a$ (respectively, $b$ and $c$ ) is the least common multiple of the local degrees of $\beta$ at each preimage of 0 (respectively, 1 and $\infty$ ). In terms of the bipartite map, $a$ is the least common multiple of the degrees of

\footnotetext{
* Partially supported by Projects Fondecyt 1150003 and Anillo ACT 1415 PIA-CONICYT

${ }^{\dagger}$ Partially supported by Projects Fondecyt 3140050 and Anillo ACT 1415 PIA-CONICYT

E-mail addresses: ruben.hidalgo@ufrontera.cl (Ruben A. Hidalgo), saul.quispe@ufrontera.cl (Saul Quispe)
} 
black vertices, $b$ is the least common multiple of the degrees of white vertices and $c$ is the least common multiple of the degrees of the faces (recall that a face of the dessin d'enfant must have an even number $2 \delta$ of boundary edges, where internal edges are counted twice; in this case $\delta$ is the degree of the face).

Two dessins d'enfant $\left(S_{1}, \beta_{1}\right)$ and $\left(S_{2}, \beta_{2}\right)$ are said to be equivalent (denoted this by the symbol $\left.\left(S_{1}, \beta_{1}\right) \sim\left(S_{2}, \beta_{2}\right)\right)$ if there is an isomorphisms $f: S_{1} \rightarrow S_{2}$ so that $\beta_{1}=\beta_{2} \circ f$. Clearly, the signature is an invariant under this equivalence relation.

There is a natural bijection between dessins d'enfants (respectively, regular dessins d'enfants), of signature $(a, b, c)$ and degree $d$, and conjugacy classes of subgroups (respectively, normal subgroups) of index $d$ of the triangular group

$$
\Delta(a, b, c)=\left\langle x, y: y^{a}=x^{b}=(x y)^{c}=1\right\rangle .
$$

By Belyi's theorem [2], each dessin d'enfant is equivalent to a dessin d'enfant $(C, \beta)$ where $C$ is an algebraic curve and $\beta$ a rational map, both defined over the field $\overline{\mathbb{Q}}$ of algebraic numbers. This provides a natural action of the absolute Galois group $\operatorname{Gal}(\overline{\mathbb{Q}} / \mathbb{Q})$ on the set of (equivalence classes of) dessins d'enfants as follows. Start with a dessin d'enfant $(C, \beta)$, defined algebraically over $\overline{\mathbb{Q}}$ and let $\sigma \in \operatorname{Gal}(\overline{\mathbb{Q}} / \mathbb{Q})$. Assume $C$ is defined by the polynomials $P_{1}, \ldots, P_{r}$ and that $\beta=Q_{1} / Q_{2}$, where all polynomials have coefficients in $\overline{\mathbb{Q}}$. Let $P_{j}^{\sigma}$ and $Q_{k}^{\sigma}$ be the polynomials obtained by applying $\sigma$ to the coefficients of $P_{j}$ and $Q_{k}$, respectively. If $C^{\sigma}$ is the algebraic curve defined by the polynomials $P_{j}^{\sigma}$ and $\beta^{\sigma}=Q_{1}^{\sigma} / Q_{2}^{\sigma}$, then $\left(C^{\sigma}, \beta^{\sigma}\right)$ still a dessin d'enfant. It is well known that the above action of the absolute Galois group is faithful [4, 5, 8, 12]. For many years, it was an open and difficult question if the absolute Galois group also acts faithfuly on the set of regular dessins d'enfants. Last year, this problem was solved by González-Diez and Jaikin-Zapirain in [6] and in a slightly weaker form by Bauer, Catanese and Grunewald in [1].

The field of moduli of a dessin d'enfant $(C, \beta)$ is the fixed field of the subgroup of $\operatorname{Gal}(\overline{\mathbb{Q}} / \mathbb{Q})$ consisting of those $\sigma$ for which $\left(C^{\sigma}, \beta^{\sigma}\right) \sim(C, \beta)$ (i.e., the field of definition of the equivalence class of the dessin d'enfant). The field of moduli is contained in any field of definition of the dessin (it is in fact the intersection of all of them by results due to Koizumi [10]), but there are examples for which the field of moduli is not a field of definition of it.

In [15], Wolfart observed that regular dessins d'enfants are definable over its field of moduli. The only explicit examples for such Galois Belyi actions were however known only for curves and dessins defined over abelian extensions of $\mathbb{Q}$. A question posed by Conder, Jones, Streit and Wolfart in [3] was if there were examples of regular dessins d'enfant with field of moduli being a non-abelian extension of $\mathbb{Q}$. In [9] Herradon answered the above positively by constructing a regular dessin d'enfant with field of moduli being $\mathbb{Q}(\sqrt[3]{2})$. Herradon starts with the following genus one non-uniform dessin d'enfant of signature $(4,6,12)$

$$
\left(C: y^{2}=x(x-1)(x-\sqrt[3]{2}), \quad \beta(x, y)=x^{3}\left(2-x^{3}\right)\right),
$$

whose field of moduli is $\mathbb{Q}(\sqrt[3]{2})$, and then he observes that its normalizing regular dessin d'enfant has the same field of moduli (he also constructs another regular dessin d'enfant with the same property, this being a quotient of the previous one).

In this paper we observe that Herradon's example belongs to a infinite family with the same property which we proceed to describe in Section 3. 


\section{Preliminaries on triangle groups}

If $l, m, n \geq 2$ are integers so that $l \leq m \leq n$ and $l^{-1}+m^{-1}+n^{-1}<1$, then the triangular group

$$
\Delta(l, m, n)=\left\langle x, y: y^{l}=x^{m}=(x y)^{n}=1\right\rangle
$$

can be seen as a discrete group of isometries of the hyperbolic plane $\mathbb{H}$, that is a triangular Fuchsian group. In this case, $\mathbb{H} / \Delta(l, m, n)$ is an orbifold of genus zero having exactly three cone points of respective orders $l, m$ and $n$. The triple $(l, m, n)$ is called the signature of $\Delta(l, m, n)$.

A triangular Fuchsian group $\Delta$ is maximal if it is not a proper subgroup of finite index of another triangle group [7]. In [13], Singerman proved that $\Delta(l, m, n)$ is maximal if and only if

$$
(l, m, n) \notin\{(l, l, l),(l, l, n),(l, m, m),(2, m, 2 m),(3, m, 3 m)\} .
$$

A Fuchsian group $\Delta$ is called non-arithmetic if the commensurate group

$$
\operatorname{Comm}(\Delta)=\left\{\gamma \in \operatorname{Aut}(\mathbb{H}):\left[\Delta: \Delta \cap \gamma \Delta \gamma^{-1}\right]<\infty,\left[\gamma \Delta \gamma^{-1}: \Delta \cap \gamma \Delta \gamma^{-1}\right]<\infty\right\}
$$

is discrete. This is not the original definition of a non-arithmetic group but it is equivalent due to a result of Margulis in [11]. The list of all the triples $(l, m, n)$ for which $\Delta$ is arithmetic has been provided by Takeuchi in [14] (there are 76 such triples):

$$
\begin{gathered}
(2,3,7),(2,3,8),(2,3,9),(2,3,10),(2,3,11),(2,3,12),(2,3,14),(2,3,16), \\
(2,3,18),(2,3,24),(2,3,30),(2,4,5),(2,4,6),(2,4,7),(2,4,8),(2,4,10), \\
(2,4,12),(2,4,18),(2,5,5),(2,5,6),(2,5,8),(2,5,10),(2,5,20),(2,5,30), \\
(2,6,6),(2,6,8),(2,6,12),(2,7,7),(2,7,14),(2,8,8),(2,8,16),(2,9,18), \\
(2,10,10),(2,12,12),(2,12,24),(2,15,30),(2,18,18),(3,3,4),(3,3,5),(3,3,6), \\
(3,3,7),(3,3,8),(3,3,9),(3,3,12),(3,3,15),(3,4,4),(3,4,6),(3,4,12), \\
(3,5,5),(3,6,6),(3,6,18),(3,8,8),(3,8,24),(3,10,30),(3,12,12),(4,4,4), \\
(4,4,5),(4,4,6),(4,4,9),(4,5,5),(4,6,6),(4,8,8),(4,16,16),(5,5,5), \\
(5,5,10),(5,5,15),(5,10,10),(6,6,6),(6,12,12),(6,24, .24),(7,7,7), \\
(8,8,8),(9,9,9),(9,18,18),(12,12,12),(15,15,15) .
\end{gathered}
$$

All the above asserts the following simple fact.

Lemma 2.1. If $p \geq 3$ is a prime integer, then $\Delta(4,2 p, 4 p)$ is maximal and non-arithmetic. In particular, $\operatorname{Comm}(\Delta(4,2 p, 4 p))=\Delta(4,2 p, 4 p)$ and, if there is a finite index subgroup $\Gamma$ of $\Delta(4,2 p, 4 p)$ and there is some $\gamma \in \operatorname{Aut}(\mathbb{H})$ so that $\gamma \Gamma \gamma^{-1} \in \Delta(4,2 p, 4 p)$, then $\gamma \in \Delta(4,2 p, 4 p)$.

Proof. It follows from the above lists that $\Delta:=\Delta(4,2 p, 4 p)$ is maximal and non-arithmetic one. The non-arithmetic property asserts that $\operatorname{Comm}(\Delta)$ is a Fuchsian triangular group containing $\Delta$; and by the maximal property, it must then follows the equality. Now, let $\Gamma$ be a finite index subgroup of $\Delta$ and let $\gamma \in \operatorname{Aut}(\mathbb{H})$ so that $\gamma \Gamma \gamma^{-1} \in \Delta$. As $\gamma \Gamma \gamma^{-1}$ is a finite index subgroup of $\gamma \Delta \gamma^{-1}$ and also of $\Delta$, and $\gamma \Gamma \gamma^{-1}<\Delta \cap \gamma \Delta \gamma^{-1}$, it follows that $\gamma \in \operatorname{Comm}(\Delta)=\Delta$. 


\section{Regular dessin d'enfants with field of moduli $\mathbb{Q}(\sqrt[p]{2})$}

Let $p \geq 3$ be a prime integer and let us consider the elliptic curve

$$
C_{0}: y^{2}=x(x-1)(x-\sqrt[p]{2}) .
$$

It is well known that the field of moduli of $C_{0}$ is $\mathbb{Q}(j(\sqrt[p]{2}))=\mathbb{Q}(\sqrt[p]{2})$, where $j$ is the elliptic modular function

$$
j(\lambda)=\left(1-\lambda+\lambda^{2}\right)^{3} / \lambda^{2}(1-\lambda)^{2} .
$$

On $C_{0}$ we consider the Belyi map

$$
\beta(x, y)=x^{p}\left(2-x^{p}\right)
$$

The dessin d'enfant $\left(C_{0}, \beta\right)$ has signature $(4,2 p, 4 p)$, which is, by Lemma 2.1 , maximal and non-arithmetic. This dessin d'enfant is non-uniform, in particular, it is non-regular (see Figure 1).

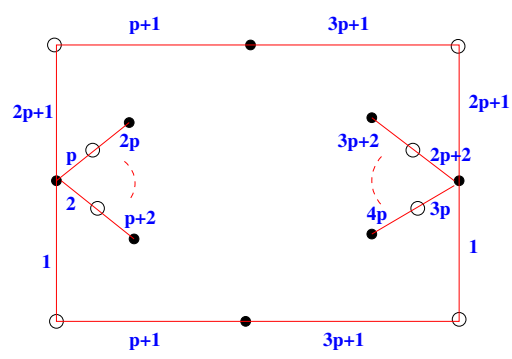

Figure 1: The dessin d'enfant $\left(C_{0}, \beta\right)$

The monodromy of the dessin $\left(C_{0}, \beta\right)$ is

$$
\begin{gathered}
\omega_{0}: \Delta=\left\langle x, y: y^{4}=x^{2 p}=(x y)^{4 p}=1\right\rangle \rightarrow \mathfrak{S}_{4 p}, \\
\omega_{0}(x)=(1,2, \ldots, p, 2 p+1,2 p+2, \ldots, 3 p)(p+1,3 p+1), \\
\omega_{0}(y) \\
\| \\
(2, p+2)(3, p+3) \cdots(p, 2 p)(2 p+2,3 p+2)(2 p+3,3 p+3) \cdots(3 p, 4 p) \\
(1,3 p+1,2 p+1, p+1),
\end{gathered}
$$

and the dessin d'enfant corresponds to the $\Delta$-conjugacy class of the subgroup

$$
F_{0}=\omega_{0}^{-1}\left(\operatorname{Stab}_{\omega_{0}(\Delta)}(1)\right)
$$

Let us set $\Gamma_{0}=\operatorname{ker}\left(\omega_{0}\right)$.

Next we list some properties of $\omega_{0}$, the first three of them are immediate from its definition. 


\section{Lemma 3.1.}

1. $\omega_{0}(x)^{p}=(1,2 p+1)(2,2 p+2) \cdots(p+1,3 p+1)$.

2. $\omega_{0}(y)^{2}=(1,2 p+1)(p+1,3 p+1)$.

3. $\omega_{0}(x y)$ has order $4 p$.

4. $\omega_{0}(\Delta)$ is a group of order $2^{2 p} p^{2}$.

5 .

$$
\prod_{j=0}^{p-1} x^{-j}\left(x^{p} y^{2}\right) x^{j} \in \Gamma_{0} .
$$

Proof. Parts (1), (2) and (3) are direct to see from the definition of $\omega_{0}$. Part (4) it is a little more difficult to see, but as we do not need it in the rest, we leave it to the reader. To check part (5) we only need to observe the following equalities:

$$
\begin{gathered}
\omega_{0}(x)^{p} \omega_{0}(y)^{2}=(2,2 p+2) \cdots(p, 3 p), \\
\omega_{0}(x)^{-1}\left(\omega_{0}(x)^{p} \omega_{0}(y)^{2}\right) \omega_{0}(x)=(1,2 p+1)(3,2 p+3) \cdots(p, 3 p), \\
\omega_{0}(x)^{-2}\left(\omega_{0}(x)^{p} \omega_{0}(y)^{2}\right) \omega_{0}(x)^{2}=(1,2 p+1)(2,2 p+2)(4,2 p+4) \cdots(p, 3 p), \\
\vdots \\
\omega_{0}(x)^{-(p-1)}\left(\omega_{0}(x)^{p} \omega_{0}(y)^{2}\right) \omega_{0}(x)^{p-1}=(1,2 p+1)(2,2 p+2) \cdots(p-1,3 p-1) .
\end{gathered}
$$

The normal subgroup $\Gamma_{0}$ corresponds to a regular dessin d'enfant $\left(\widetilde{C}_{0}, \widetilde{\beta}_{0}\right)$ with signature $(4,2 p, 4 p)$. As the previous signature is maximal (by Lemma 2.1), we have that

$$
\operatorname{deck}\left(\widetilde{\beta}_{0}\right)=\operatorname{Aut}\left(\widetilde{C}_{0}\right) \cong \omega_{0}(\Delta) .
$$

Also, as a consequence of the Riemann-Hurwitz formula, the genus of $\widetilde{C}_{0}$ is

$$
g_{p}=1+3 \times 2^{2 p-3} p(p-1) .
$$

The Galois orbit of $\left(C_{0}, \beta\right)$ is given by the $p$ dessins d'enfants (see Figure 2)

$$
\left(C_{k}, \beta\right) ; \quad k=0,1, \ldots, p-1
$$

where

$$
C_{k}: y^{2}=x(x-1)\left(x-\rho_{p}^{k} \sqrt[p]{2}\right), \quad \rho_{p}=e^{2 \pi i / p},
$$

whose monodromy $\omega_{k}: \Delta \rightarrow \mathfrak{S}_{4 p}$ is defined by

$$
\begin{gathered}
\omega_{k}(x) \\
\| \\
(1,2, \ldots, k+1,2 p+k+2, \ldots, 3 p, 2 p+1,2 p+2, \ldots, 2 p+k+1, k+2, \ldots, p) \\
(p+k+1,3 p+k+1),
\end{gathered}
$$


and

$$
\begin{gathered}
\omega_{k}(y) \\
\| \\
(2, p+2)(3, p+3) \cdots(p, 2 p)(2 p+2,3 p+2)(2 p+3,3 p+3) \cdots(3 p, 4 p) \\
(1,3 p+1,2 p+1, p+1),
\end{gathered}
$$

and the dessin d'enfant corresponds to the $\Delta$-conjugacy class of the subgroup

$$
F_{k}=\omega_{k}^{-1}\left(\operatorname{Stab}_{\omega_{k}(\Delta)}(1)\right) .
$$

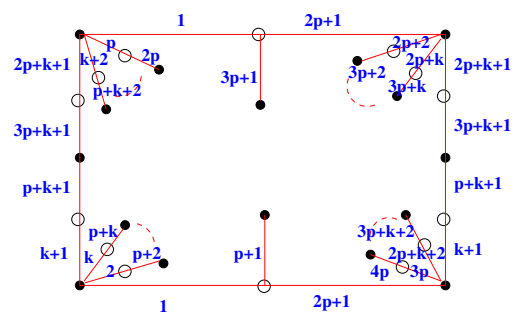

Figure 2: The dessin d'enfant $\left(C_{k}, \beta\right)$

The normal subgroup $\Gamma_{k}=\operatorname{ker}\left(\omega_{k}\right)$ corresponds to a regular dessin d'enfant $\left(\widetilde{C}_{k}, \widetilde{\beta}_{k}\right)$ with signature $(4,2 p, 4 p)$. Again, by maximality of the signature,

$$
\operatorname{deck}\left(\widetilde{\beta}_{k}\right)=\operatorname{Aut}\left(\widetilde{C}_{k}\right) \cong \omega_{k}(\Delta) \cong \omega_{0}(\Delta) .
$$

Theorem 3.2. The field of moduli of $\left(\widetilde{C}_{0}, \widetilde{\beta}_{0}\right)$ is $\mathbb{Q}(\sqrt[p]{2})$.

Proof. As the regular dessin d'enfant $\left(\widetilde{C}_{k}, \widetilde{\beta}_{k}\right)$ is the normalization of the dessin d'enfant $\left(C_{k}, \beta\right)$, we see that the Galois orbit of $\left(\widetilde{C}_{0}, \widetilde{\beta}_{0}\right)$ is given by the following $p$ dessins d'enfants

$$
\left(\widetilde{C}_{k}, \widetilde{\beta}_{k}\right) ; \quad k=0,1, \ldots, p-1 .
$$

It follows that the field of moduli of $\left(\widetilde{C}_{0}, \widetilde{\beta}_{0}\right)$ is a subfield of $\mathbb{Q}(\sqrt[p]{2})$. As $\mathbb{Q}(\sqrt[p]{2})$ is an extension of degree $p$ (a prime integer) of $\mathbb{Q}$, in order to see that the field of moduli is exactly $\mathbb{Q}(\sqrt[p]{2})$ we only need to check that $\Gamma_{0}$ and $\Gamma_{1}$ are not conjugated in Aut $(\mathbb{H})$. As $\Gamma_{0}$ is a finite index subgroup of the maximal and non-arithmetic group $\Delta(4,2 p, 4 p)$, it follows, from Lemma 2.1, that we only need to check that $\Gamma_{0} \neq \Gamma_{1}$. This last can be noted by part (5) of Lemma 3.1 and the fact that $\prod_{j=0}^{p-1} x^{-j}\left(x^{p} y^{2}\right) x^{j} \notin \Gamma_{1}$; since

$$
\prod_{j=0}^{p-1} \omega_{1}(x)^{-j}\left(\omega_{1}(x)^{p} \omega_{1}(y)^{2}\right) \omega_{1}(x)^{j}=(p+1,3 p+1)(p+2,3 p+2) .
$$

This last can be checked by observing that

$$
\omega_{0}(x)^{p}=\omega_{1}(x)^{p}(p+1,3 p+1)(p+2,3 p+2),
$$




$$
\begin{gathered}
\omega_{1}(y)^{2}=\omega_{0}(y)^{2}, \\
\omega_{1}(x)^{p} \omega_{1}(y)^{2}=\left(\omega_{0}(x)^{p} \omega_{0}(y)^{2}\right)(p+1,3 p+1)(p+2,3 p+2),
\end{gathered}
$$

and, for $j=1, \ldots, p-1$,

$$
\begin{gathered}
\omega_{1}(x)^{-j}\left(\omega_{1}(x)^{p} \omega_{1}(y)^{2}\right) \omega_{1}(x)^{j} \\
\| \\
\omega_{0}(x)^{-j}\left(\omega_{0}(x)^{p} \omega_{0}(y)^{2}\right) \omega_{0}(x)^{j}(p+1,3 p+1)(p+2,3 p+2) .
\end{gathered}
$$

\section{Acknowledgments}

The authors are deeply grateful to the referee for his/her valuable suggestions/corrections to the previous version of this paper.

\section{References}

[1] I. Bauer, F. Catanese and F. Grunewald, Faithful actions of the absolute Galois group on connected components of moduli spaces, Invent. Math. 199 No. 3 (2015), 859-888, doi: 10.1007/s00222-014-0531-2.

[2] G. V. Bely̌̌, On Galois extensions of a maximal cyclotomic field, Izv. Akad. Nauk SSSR Ser. Mat. 43 (1979) 267-276.

[3] M. Conder, G. Jones, M. Streit, J. Wolfart, Galois actions on regular dessins of small genera, Rev. Mat. Iberoam. 29 No. 1 (2013), 163-181, doi: 10.4171/RMI/717.

[4] E. Girondo and G. González-Diez, Introduction to compact Riemann surfaces and dessins d'enfants, London Mathematical Society Student Texts 79. Cambridge University Press, Cambridge, 2012.

[5] E. Girondo and G. González-Diez, A note on the action of the absolute Galois group on dessins, Bull. London Math. Soc. 39 No. 5 (2007), 721-723, doi: 10.1112/blms/bdm035.

[6] G. Gonzaléz-Diez and A. Jaikin-Zapirain, The absolute Galois group acts faithfully on regular dessins and on Beauville surfaces, Proc. London Math. Soc. 111 No. 4 (2015), 775-796, doi: 10.1112/plms/pdv041.

[7] L. Greenberg, Maximal Fuchsian groups, Bull. Amer. Math. Soc. 69 No. 4 (1963), 569-573, doi: 10.1090/S0002-9904-1963-11001-0.

[8] A. Grothendieck, Esquisse d'un programme, in: Geometric Galois Actions. 1. Around Grothendieck's Esquisse d'un programme. Ed. L. Schneps and P. Lochak, pp. 5-48, London Math. Soc. Lecture Note Ser. 242, Cambridge University Press (1997).

[9] M. Herradon, An explicit quasiplatonic curve with non-abelian moduli field, Revista Matematica Complutense 29 No.3 (2016), 725-739, doi: 10.1007/s13163-016-0196-z.

[10] S. Koizumi, Fields of moduli for polarized Abelian varieties and for curves, Nagoya Math. J. 48 (1972), 37-55.

[11] G. Margulis, Discrete subgroups of semisimple Lie groups. Springer-Verlag, 1991, doi: 10.1007/978-3-642-51445-6.

[12] L. Schneps, Dessins d'enfant on the Riemann sphere, in: The Grothendieck theory of dessins d'enfants. Edited by Leila Schneps. London Math. Soc. Lecture Note Ser. 200. Cambridge University Press, Cambridge, 1994, 47-77. 
[13] D. Singerman, Finitely maximal Fuchsian groups, J. London Math. Soc. (2) 6 (1972), 29-38, doi: $10.1112 / \mathrm{jlms} / \mathrm{s} 2-6.1 .29$.

[14] K. Takeuchi, Arithmetic triangle groups, J. Math. Soc. Japan 29 No. 1 (1977), 91-106, doi: 10.2969/jmsj/02910091.

[15] J. Wolfart, $A B C$ for polynomials, dessins d'enfants and uniformization - a survey. Elementare und analytische Zahlentheorie, 313-345, Schr. Wiss. Ges. Johann Wolfgang Goethe Univ. Frankfurt am Main, 20, Franz Steiner Verlag Stuttgart, Stuttgart, 2006. 